\title{
Etching and ellipsometry studies on CL-VPE grown GaN epilayer
}

\author{
P. PUVIARASU* \\ Department of Physics, PSG College of Technology, Peelamedu, Coimbatore-641004, India
}

\begin{abstract}
The surface morphological characteristics of wet chemical etched GaN layers grown at different temperatures on $\left(\begin{array}{llll}0 & 0 & 0 & 1\end{array}\right)$ sapphire substrates by Chloride-Vapor Phase Epitaxy (Cl-VPE) have been studied using optical microscope. Significant surface morphology changes have been observed in correlation to the growth temperature and etching time. Also optical properties of the as grown and high-energy silicon ( $\mathrm{Si}$ ) ion irradiated gallium nitride $(\mathrm{GaN})$ epilayers were studied using monochromatic ellipsometry. The effect of ion fluences on the refractive index of the GaN has been investigated and it has been found to decrease with an increase of ion fluence. This decrease is attributed to irradiation-induced defects and polycrystallization which plays an important role in determining the optical properties of silicon ( $\mathrm{Si}$ ) ion irradiated GaN layers.
\end{abstract}

Keywords: gallium nitride; vapor phase epitaxy, acid etching; optical microscope; ellipsometry

(C) Wroclaw University of Science and Technology.

\section{Introduction}

Group III-nitrides have been considered as a promising system for semiconductor device applications in blue and ultraviolet (UV) wavelengths and, just like their highly successful arsenic and phosphorous-based cousins, have been exploited in the infrared (IR), red and green wavelengths [1,2]. Currently, considerable attention has been paid to GaN-based compound semiconductors due to their potential applications in short wavelength (blue and UV) light emitting diodes (LEDs), laser diodes (LDs) and UV-photodetectors [3]. Gallium nitride and related alloys are the subject of extensive research because of their potential application in blue lasers, high power and high frequency electronics [4]. The defect density, especially the threading dislocation density, plays a critical role in performances of electronic devices [5].

Wet chemical etching, a common technique used for surface defect characterization, appears to be less reliable for GaN. Compared to other etchants $(\mathrm{NaOH}, \mathrm{KOH})$, hot $(433 \mathrm{~K}) \mathrm{H}_{3} \mathrm{PO}_{4}$ etches $\mathrm{N}$-polar GaN films very quickly resulting either

*E-mail: puvi_au@yahoo.co.in in complete removal or a drastic change in the surface morphology as revealed by optical microscope [7]. Refractive index values of $\mathrm{GaN}$, obtained by various optical methods, have been reported in the literature [8]. Ellipsometry is a classical and precise method for determining the optical constants, thickness and nature of reflecting surfaces or films formed on them.

In this paper, we report a systematic investigation on the use of hot phosphoric acid etching of GaN samples grown at different temperatures on sapphire substrate by Chloride-Vapour Phase Epitaxy (Cl-VPE). The surface morphology of the Cl-VPE grown GaN etched samples has been examined using Leitz Wetzlar metallurgical microscope. The effects of Si ion irradiation on the refractive index and on the thickness of GaN layers irradiated with different fluences were also characterized. Gaertner Research Ellipsometer L119 XUV has been used to calculate the refractive index and thickness of the $\mathrm{Si}$ irradiated $\mathrm{GaN}$. A He$\mathrm{Ne}$ laser with a wavelength of $632.8 \mathrm{~nm}$ has been used for the refractive index measurements. Two parameters, $\Delta$ and $\Psi$, were obtained in ellipsometry studies as a function of wavelength. These two parameters are related to the complex reflection ratio $\rho$ by: 


$$
\rho=\frac{R_{p}}{R_{S}}=\tan (\Psi) \cdot e^{i \Delta}
$$

where $R_{p}$ and $R_{s}$ are complex amplitude reflection coefficients of the light polarized parallel and perpendicular to the incidence plane, respectively. The dispersions of refractive indices and film thickness were determined by fitting experimental data of $\Delta$ and $\Psi$ using a suitable model. The film thickness, d, obtained by fitting to the ellipsomery data, together with the order $\mathrm{m}$ of the interference fringes, was then used to obtain refined values of refractive indices from the relationship [9]:

$$
n=\frac{m \lambda}{2 d}
$$

\section{Experimental}

A horizontal chloride vapor phase reactor has been employed, where $\mathrm{GaCl}_{3}$ and $\mathrm{NH}_{3}$ have been used as $\mathrm{Ga}$ and $\mathrm{N}$ precursors, respectively. $\mathrm{GaCl}_{3}$ was transported in the reactor using $\mathrm{N}_{2}$ as the carrier gas. The gallium nitride $(\mathrm{GaN})$ films were deposited on $\left(\begin{array}{llll}0 & 0 & 0 & 1\end{array}\right) \mathrm{Al}_{2} \mathrm{O}_{3}$ substrate, as discussed in the literature [10]. The growth of GaN films was carried out at the growth temperatures of $1243 \mathrm{~K}$ and $1263 \mathrm{~K}$ at a fixed flow rate (2 SLM) of the ammonia gas. Etching of GaN samples was performed in a glass container in $\mathrm{H}_{3} \mathrm{PO}_{4}(85 \%)$ at $433 \mathrm{~K}$. The acid container was placed on a hot plate and a temperature probe was immersed in the acid for temperature control. The acid solution was stirred for temperature uniformity, and the temperature was controlled within $\pm 1{ }^{\circ} \mathrm{C}$. After the acid reached a specified temperature, the GaN samples were immersed in the acid for two periods of $5 \mathrm{~min}$ and $10 \mathrm{~min}$ and immediately taken out of the acid and quenched in deionized water to stop further etching.

The GaN layers were cut in samples of $5 \mathrm{~mm} \times 5 \mathrm{~mm}$ and were used for irradiation. Before irradiation the layers were etched with dilute $\mathrm{HCl}$ to remove surface oxides. $80 \mathrm{MeV}$ silicon ion irradiation has been carried out on $\mathrm{GaN}$ epilayers at room temperature using 16-MV Pelletron Tandem Accelerator (NSC) under a background vacuum pressure of $400 \mathrm{~Pa}$ at various doses ranging from $1 \times 10^{11} \mathrm{~cm}^{-2}$ to $1 \times 10^{14} \mathrm{~cm}^{-2}$. In order to find the effect of ion fluences on the refractive indices, thickness and extinction coefficient of the GaN layers, the irradiated samples have been analyzed using ellipsometric measurements.
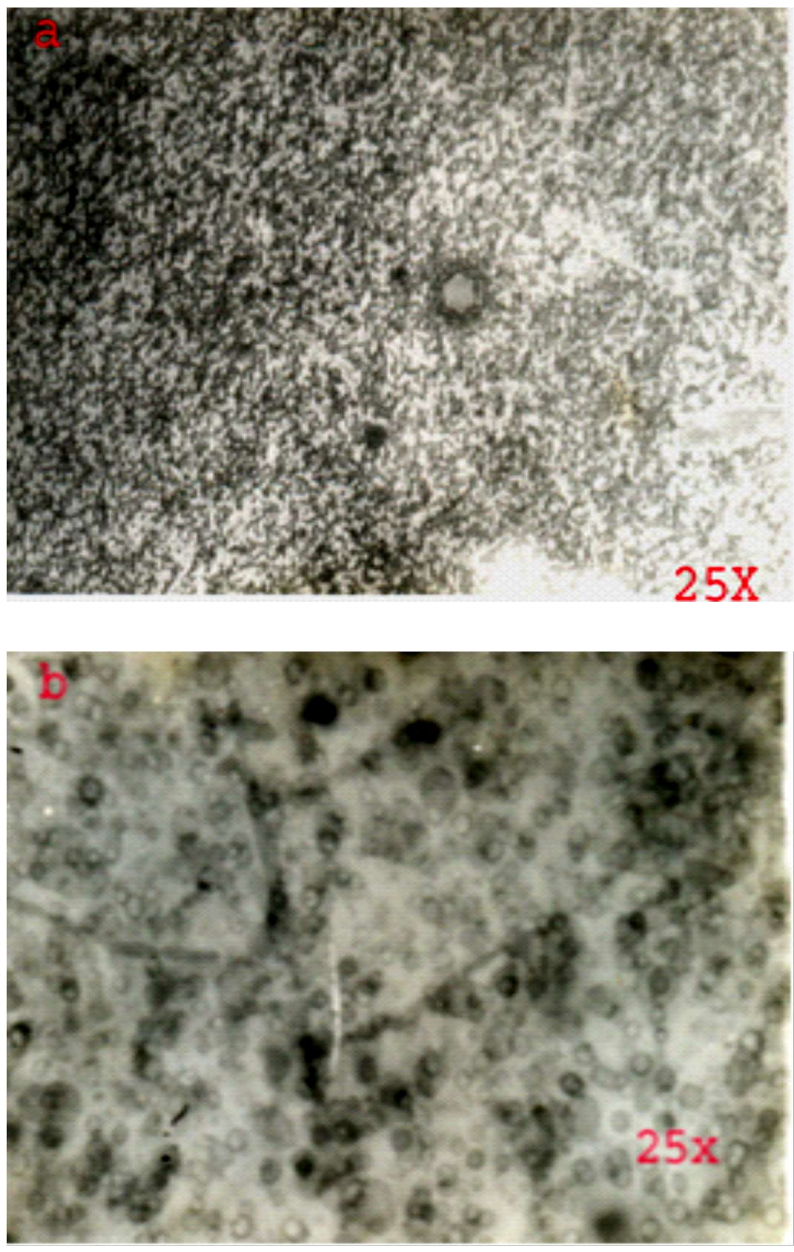

Fig. 1. Optical images of a Cl-VPE (1243 K) GaN grown on sapphire substrate after etching in $\mathrm{H}_{3} \mathrm{PO}_{4}$ at $433 \mathrm{~K}$ (a) for $5 \mathrm{~min}$ and (b) for $10 \mathrm{~min}$.

\section{Results and discussion}

Etch pits can be formed at point-defect clusters or dislocations. Thus, the process of etch pit formation may be viewed in terms of two aspects: thermodynamic and kinetic. The thermodynamic aspect is associated with the free energy change involved, and deals with the rate of etchpit nucleation. The kinetic aspect, connected with 
the lateral motion of steps, involves mutual interaction between steps and absorption of impurities at them. According to the Burgers vectors of threading dislocations in a $\mathrm{GaN}$ epilayer, pure edge threading dislocations emerge in the terraces, while screw/mixed threading dislocations are associated with the step terminations [11]. Fig. 1 shows the optical microscope images of $1243 \mathrm{~K}$ grown $\mathrm{GaN}$ sample etched at $433 \mathrm{~K}$ for $5 \mathrm{~min}$ and $10 \mathrm{~min}$, respectively.
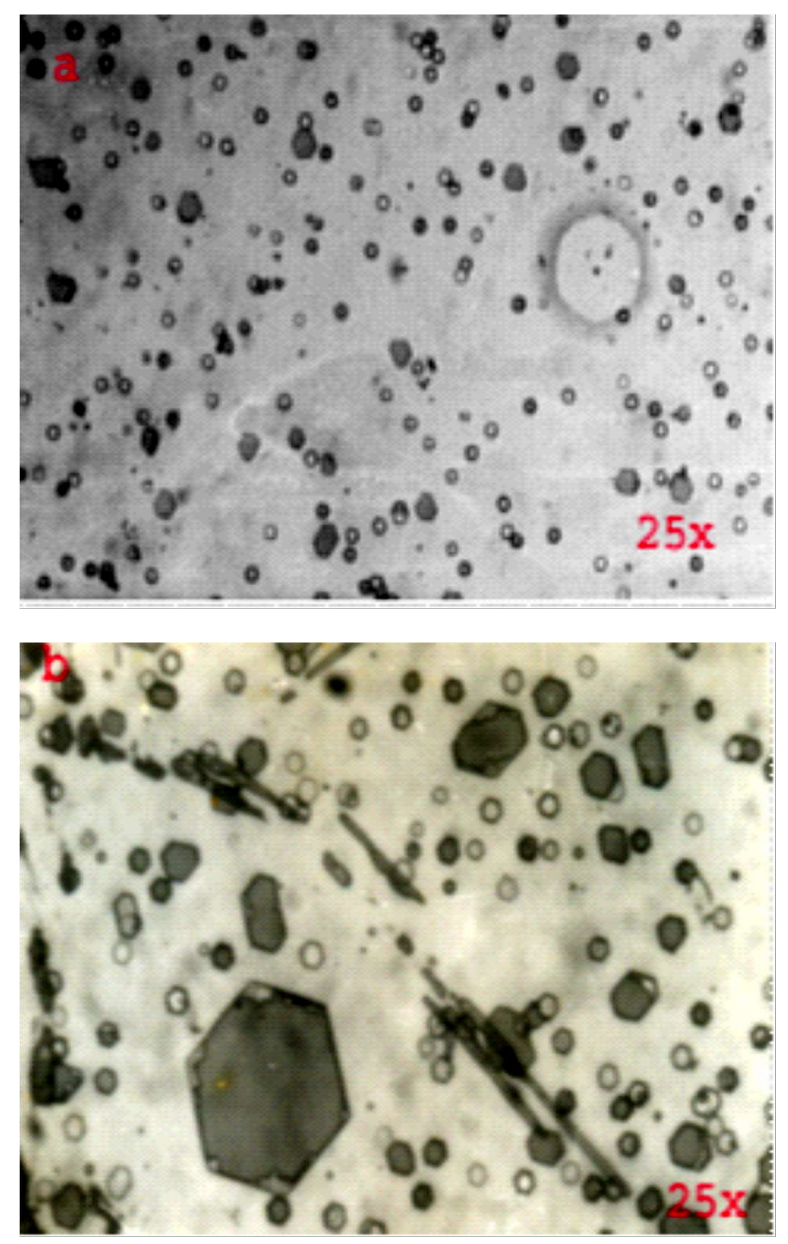

Fig. 2. Optical images of a Cl-VPE (1263 K) GaN grown on sapphire substrate after etching in $\mathrm{H}_{3} \mathrm{PO}_{4}$ at $433 \mathrm{~K}$ (a) for $5 \mathrm{~min}$ and (b) for 10 $\min$.

The increase in the size of the pits and formation of new additional pits have been observed, when the samples were allowed for more etching time. The density of etch pits was about
$7.32 \times 10^{5} \mathrm{~cm}^{-2}$. Fig. 2 shows the etched GaN samples grown at $1263 \mathrm{~K}$. The etch pits distribution indicates two types of pits: first type is relatively small and circular in shape, while the other one is of large hexagonal nature. The origin of the large hexagonal pits is due to both pure edge and edge screw mixed dislocations and the small represent edge dislocations [12]. While increasing the etch time, the pit size increased due to coalescence of neighboring pits. The density of etch pits in the sample grown at high temperature is $3.2 \times 10^{5} \mathrm{~cm}^{-2}$. Lin et al. [13] reported the refractive index of wurtzite GaN film grown by plasmaenhanced molecular beam epitaxy as 2.78. In our Chloride-Vapor Phase Epitaxy we measured the refractive index of wurtzite $\mathrm{GaN}$ as 1.36. This decrease in the refractive index value is due to the growth defects involved in the GaN films.

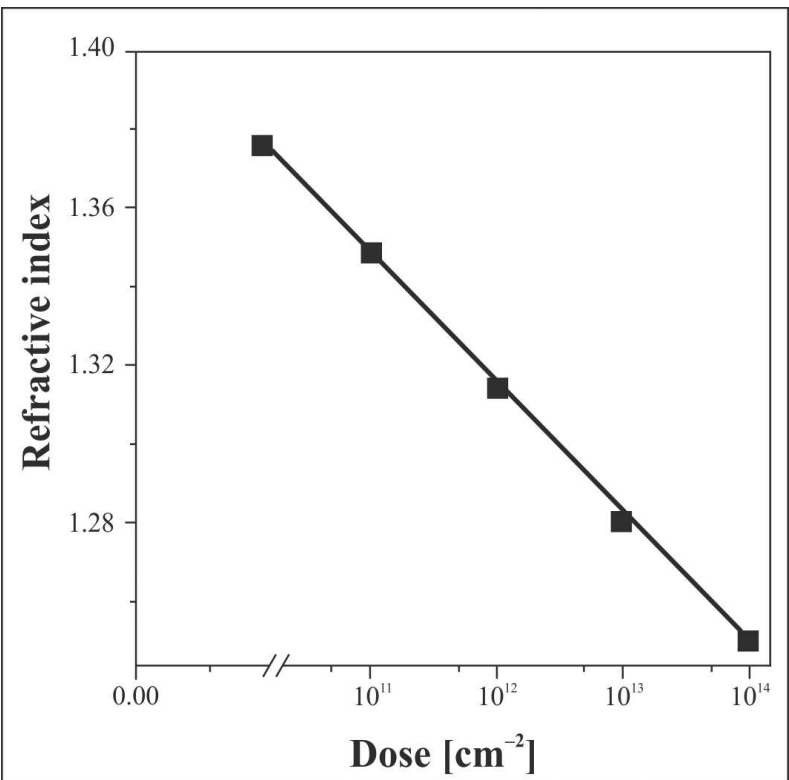

Fig. 3. Refractive index of as-grown and high energy $\mathrm{Si}$ ion irradiated $\mathrm{GaN}$.

Fig. 3 shows the refractive indices of the $\mathrm{Cl}$ VPE grown GaN layers and the high energy irradiated GaN layers. The refractive index of the as-grown $\mathrm{GaN}$ epilayer is 1.36 at room temperature. The refractive indice value gradually decreases up to 1.25 for the fluence of $10^{14} \mathrm{~cm}^{-2}$ upon Si irradiation. The radiation-induced disorder in $\mathrm{GaN}$ layer creates defects that recrystallize into 


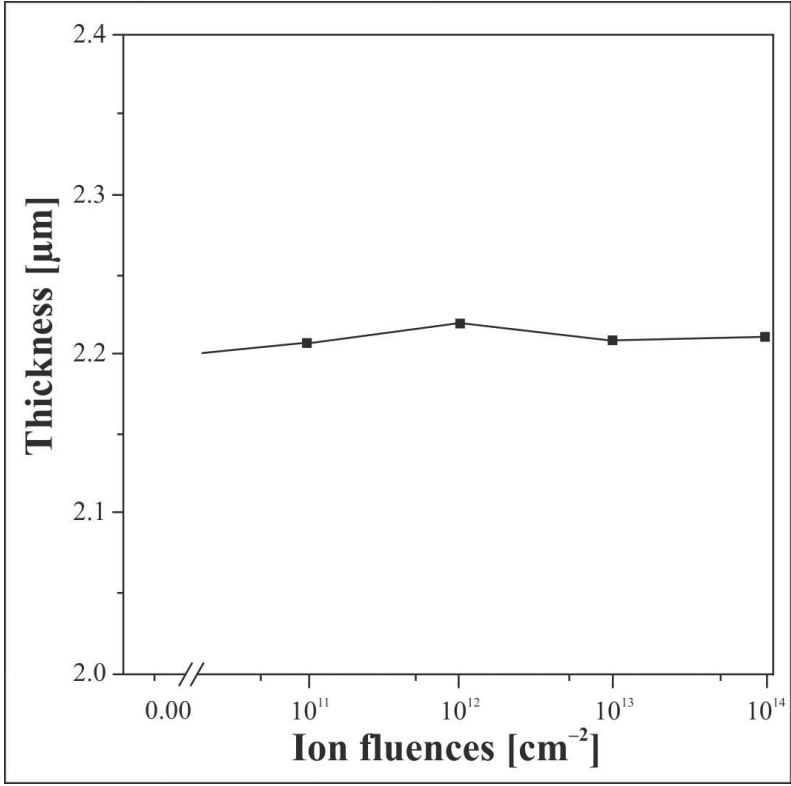

Fig. 4. Effect of Si ion irradiation on the film thickness of the GaN layers.

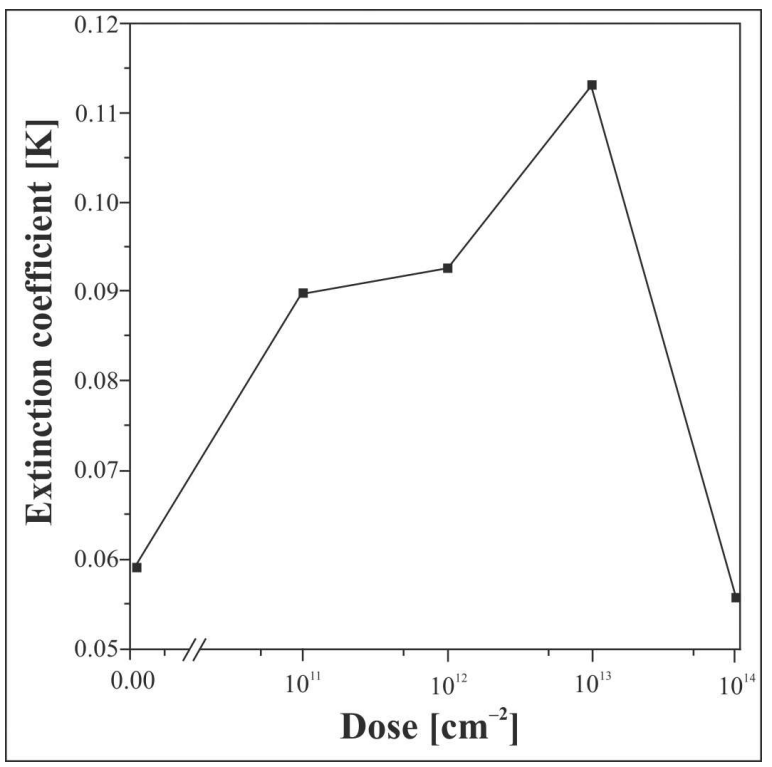

Fig. 5. Extinction coefficient of as-grown and high energy Si ion irradiated GaN layers.

polycrystalline grains of GaN. The structure of the damages produced by electronic excitations depends on the ion fluence. At small fluences only point defects and point defect complexes are detected. With increasing ion fluence, discontinuous tracks and subsequently amorphous layers are formed. An often used description of track formation in solids is based on the thermal spike model. In this model, it is assumed that the material melts along the ion trajectory up to the depths for which the electronic energy loss exceeds a certain critical value followed by fast cooling and resolidification. Due to ion irradiation, GaN is damaged and recrystallized into polycrystalline grains of $\mathrm{GaN}$ [14]. It was observed that during irradiation, the irradiated layer of $\mathrm{GaN}$ may be amorphorized to some extent due to the generated defects. These grains would decrease the refractive index of the GaN layers.

The effect of high energy irradiation on the thickness of the GaN films is shown in Fig. 4. No big change has been observed in the thickness of the high energy Si ion irradiated GaN films. However, the results show that due to heavy ion irradiation, the optical properties of the Cl-VPE grown layers have changed drastically as compared with the GaN film.

Fig. 5 shows the extinction coefficient of asgrown and high energy $\mathrm{Si}$ ion irradiated $\mathrm{GaN}$. The extinction coefficient of the as-grown $\mathrm{GaN}$ epilayer is 0.0587 at room temperature. The extinction coefficient value gradually increases up to 0.1129 for the fluence of $10^{13} \mathrm{~cm}^{-2}$ and gradually decreases up to 0.0556 for the fluence of $10^{14} \mathrm{~cm}^{-2}$ upon $\mathrm{Si}$ irradiation.

\section{Conclusions}

In summary, we have systematically evaluated hot phosphoric-acid etching of various $\mathrm{GaN}$ samples grown at different temperatures. Acid etching is an effective method for evaluating the type and density of dislocations in GaN. The optimal etch condition, however, depends on the film deposition technique and film quality. Evaluation of the refractive index of the as-grown and $\mathrm{Si}$ ion irradiated $\mathrm{Cl}-\mathrm{VPE}$ grown $\mathrm{GaN}$ on sapphire substrate has been made. The studies revealed that the refractive index decreases with an increase in ion fluence. The decreased refractive indices are attributed to irradiation-induced defects and polycrytallization. 
However, no change has been observed in the thickness of the layer. Evaluation of the extinction coefficient of the as grown and Si ion irradiated Cl-VPE grown GaN on sapphire substrate has been made. The extinction coefficient gradually increases and then decreases with an increase in ion fluence.

\section{References}

[1] Nakamura S., Iwasa N., Seno M., Mukai T., Jpn. J. Appl. Phys., 31 (1992), 1258.

[2] Galagurov L., Chong P S., Appl. Phys. Lett., 68 (1996), 43.

[3] Shokhovets S., Gadhahn R., Gobsch G., Cheng T.S., FOXON C.T., KIPSHIDZE G.D., RICHTOR W., J. Appl. Phys., 86 (1999), 2602.

[4] Minutess Ky M.S., White M., Hu E.L., Appl. Phys. Lett., 68 (1996), 1531.

[5] Zolper J.C., Wilson R.G., Pearton S.J., Appl. Phys. Lett., 68 (1996), 1945.

[6] Hong S.K., YaO T., Kim B.J., Appl. Phys. Lett., 77 (2000), 82
[7] Weyher J.C., Muller S., Grzegory I., Porowski S., J. Cryst. Growth, 182 (1997) 17.

[8] Yu G., Wang G., Ishikawa H., Umeno M., Soga T., Egawa T., Watanabe J., Jimbo T., Appl. Phys. Lett., 70 (1997), 24.

[9] Lian C.X., Li X.Y., LiU J., Semicond. Sci. Technol., 19 (2004), 417.

[10] Varadarajan E., Puviarasu P., Kumar J., Dhanasekaran R., J. Cryst. Growth, 260 (2004), 43.

[11] Chen J., Wang J.F., Wang H., Zhu J.J., Zhang S.M., ZHAO D.G., JiANG D.C., YANG H., JHAN U., Ploog K.H., Semicond. Sci. Technol., 21 (2006), 1229.

[12] Wen T.C., Lee W.I., Sheu J.K., Chi G.C., Solid State Electron., 46 (2002), 555.

[13] Lin M.E., Sverdlov B.N, Strite S., Morkoc H., DRAKIN A.E., Electron. Lett., 29 (1993) 1759.

[14] Herre O., Wendler E., Gaiduk P.I., Komarov F.F., Klaumunzer S., Meier P., Phys. Rev. B, 8 (1998), 58

Received 2015-05-06

Accepted 2017-01-05 